\title{
Impacto de alterações ambientais na ecologia de flebotomíneos no sul do Brasil
}

\author{
Impact of environmental changes on sand fly \\ ecology in southern Brazil
}

Ueslei Teodoro ${ }^{1}$

João B. Kühl 1

Demilson R. dos Santos 2

Élcio S. dos Santos 2

\footnotetext{
${ }^{1}$ Departamento de Análises Clínicas, Universidade Estadual de Maringá. Av. Colombo 5790, Maringá, PR, 87020-900, Brasil. ueslei@wnet.com.br 2 Fundação Nacional de Saúde/Subdistrito de Maringá Ministério da Saúde. Av. Gastão Vidigal 55, Maringá, $P R$ 87050-040, Brasil.
}

Abstract Tree felling around a chicken coop and periodic insecticide spraying at the Palmital farm in the county of Terra Boa in southern Brazil impacted the ecology of sand fly species. The most prevalent species (51.6\%) before clearing the trees and insecticide spraying was Lutzomyia migonei (França, 1920), and it is now Lutzomyia (Nyssomyia) whitmani (Antunes \& Coutinho, 1939) (89.8\%). There was a highly significant difference between the proportions of L. whitmani reported by this paper (89.8\%) and another publication before the tree felling and insecticide spraying (32.6\%).

Key words Psychodidae; Vectors' Ecology; Disease Vectors; Leishmaniasis

Resumo Verificou-se que a abertura de uma clareira ao redor de um galinheiro e aplicações periódicas de inseticidas refletiram na ecologia das espécies de flebotomíneos na Fazenda Palmital, município de Terra Boa, Estado do Paraná, sul do Brasil. A espécie dominante (51,6\%) antes do desmatamento e das aplicações de inseticidas era Lutzomyia migonei (França, 1920). Após estas medidas, Lutzomyia (Nyssomyia) whitmani (Antunes \& Coutinho, 1939) passou a predominar (89,8\%). A diferença de proporções de L. whitmani nesta investigação (89,8 \%) em relação à outra (32,6\%), realizada antes da abertura da clareira e das aplicações de inseticidas, foi altamente significativa.

Palavras-chave Psychodidae; Ecologia de Vetores; Vetores de Doenças; Leishmaniose 


\section{Introdução}

A leishmaniose tegumentar vem se mostrando endêmica no Estado do Paraná (Roberto et al., 1997) e alguns estudos sobre flebotomíneos têm sido realizados visando o conhecimento dos hábitos destes insetos e alternativas para o seu controle (Teodoro, 1995; Teodoro \& Kühl, 1997; Teodoro et al., 1993a; 1993b, 1998a).

O predomínio de Lutzomyia (Nyssomyia) whitmani (Antunes \& Coutinho 1939) sobre outras espécies de flebotomíneos tem sido constatado em distintos ecótopos artificiais no norte do Estado do Paraná (Cat et al., 1974; Teodoro, 1995; Teodoro et al., 1993b, 1998b). Contudo, investigações realizadas também em ambientes antropogênicos nos municípios de Jussara (Aguiar et al., 1989) e São Jorge do Ivaí (Teodoro \& Kühl, 1997) apontaram Lutzomyia (Nyssomyia) intermedia (Lutz \& Neiva, 1912) como espécie dominante. No município de Terra Boa, Lutzomyia migonei (França, 1920) prevaleceu sobre as demais espécies de flebotomíneos em apenas um dos ecótopos onde foram feitas as coletas (Teodoro et al., 1993b). Os municípios de Jussara, São Jorge do Ivaí e Terra Boa localizam-se no norte do Paraná e fazem fronteira entre si.

Neste trabalho, procurou-se verificar os efeitos do desmatamento e de desinsetizações periódicas nas margens da mata sobre a densidade populacional, a biodiversidade e a relação de dominância entre as espécies de flebotomíneos. Para tanto, compararam-se os resultados de coletas destes insetos em dois períodos distintos em uma mesma localidade do sul do Brasil.

\section{Material e métodos}

A área onde foi desenvolvida a pesquisa compreende uma mata residual alterada, na Fazenda Palmital, município de Terra Boa, no norte do Estado do Paraná, a 23o45' S e 52o26’ W (Figura 1). A vegetação nativa era constituída de mata densa tropical de transição para subtropical, com queda parcial das folhas de algumas espécies no inverno. O clima é tropical de transição para subtropical, apresentando temperaturas médias anuais em torno de $23^{\circ} \mathrm{C}$, com invernos brandos e períodos de seca hibernal pouco pronunciados em julho e agosto. A temperatura média máxima anual atingiu $30,80 \mathrm{C}$ em 1992, e a média mínima, 15,60 C em 1988 (Figura 2). Nos dias mais quentes do ano, a temperatura pode chegar a $41^{\circ} \mathrm{C}$ e, nos dias mais frios, atinge temperaturas negativas. A precipi- tação anual de 1988 a 1997 na Fazenda Palmital variou de $1.168 \mathrm{~mm}$ em 1988 a $2.216,4 \mathrm{em}$ 1997, com média anual de 1.766,1 mm (Figura 2). Neste período, o mês mais chuvoso foi janeiro, com média de $289,7 \mathrm{~mm}$, e os mais secos, julho, com média de 59,2 mm, e agosto, com 71,1 mm. Os dados sobre as temperaturas e precipitações pluviométricas registradas na Fazenda Palmital foram fornecidos pela Cia. Melhoramentos Norte do Paraná (Figura 2). Nas áreas desmatadas da Fazenda Palmital, o solo vem sendo ocupado especialmente com o plantio de soja, milho e trigo. A região onde esta área se insere foi descrita anteriormente de forma mais detalhada (Teodoro, 1995).

De janeiro a dezembro de 1997 foram feitas oito coletas mensais de flebotomíneos, duas vezes por semana, das $21 \mathrm{~h}$ às $24 \mathrm{~h}$, perfazendo um total de 288 horas. As coletas foram feitas com uma armadilha luminosa de Falcão (1981) instalada dentro de um galinheiro (G) (Figura 1), na Fazenda Palmital. Aliás, o mesmo galinheiro utilizado em pesquisa anterior por Teodoro et al. (1993b), com coletas realizadas também com armadilha de Falcão, entre $21 \mathrm{~h}$ e $3 \mathrm{~h}$, totalizando 216 horas, de novembro de 1988 a abril de 1990 .

A média horária (MH) de flebotomíneos coletados de janeiro a dezembro de 1997 foi comparada com a obtida de novembro de 1988 a abril de 1990 (Tabela 1). No espaço de tempo entre estes dois períodos foram cortadas inúmeras árvores para a abertura de uma clareira em volta do galinheiro G (Figura 1). Desta forma, o ambiente ficou mais exposto à luz solar, diminuindo o teor de umidade do solo. Além disso, periodicamente, têm sido feitas desinsetizações nas margens da mata, com pulverizador Jacto ${ }^{\circledR}$ PJ 600, usando-se inseticidas à base de piretróides, dissolvidos em água, conforme solicitação dos moradores da Fazenda Palmital, ao detectarem o aumento da população de flebotomíneos no peridomicílio. Ressalta-se que as desinsetizações, que vinham sendo feitas na década de 80 , foram interrompidas de novembro de 1988 a abril de 1990, sendo retomadas a partir de então.

Segundo Marcondes (1996) e Marcondes et al. (1998), a espécie que tem sido referida no Estado do Paraná como Lutzomyia (Nyssomyia) intermedia (Lutz \& Neiva, 1912) (Aguiar et al, 1989; Consolim et al., 1990; Teodoro, 1995; Teodoro \& Kühl, 1997; Teodoro et al., 1993a; 1993b), é, na verdade, Lutzomyia neivai (Pinto, 1926). Por esta razão adotamos esta nomenclatura no presente trabalho. 


\section{Figura 1}

Coordenadas do município de Terra Boa e local de instalação da armadilha de Falcão em ambiente antrópico, na Fazenda Palmital, Município de Terra Boa, Estado do Paraná, Brasil.
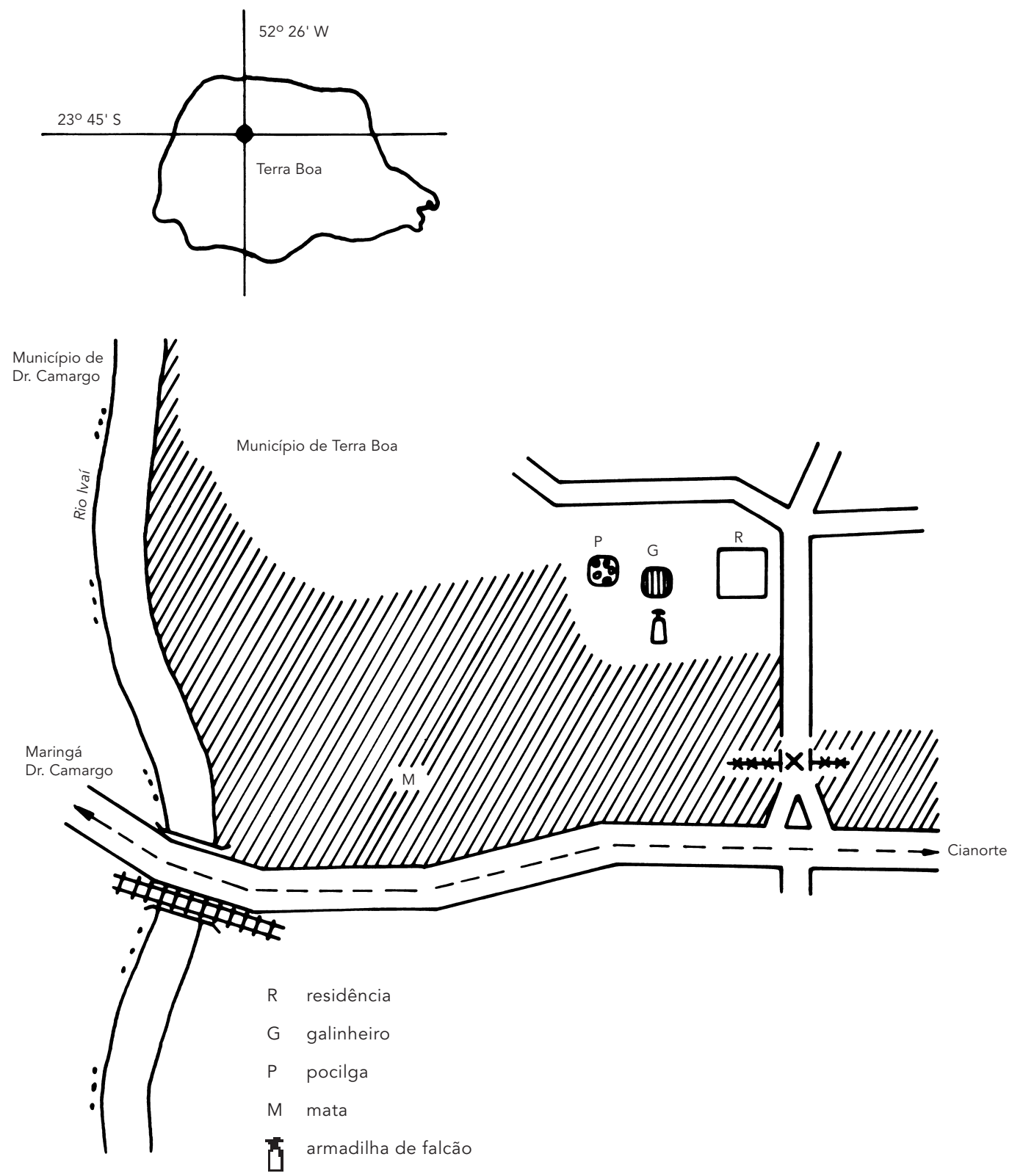


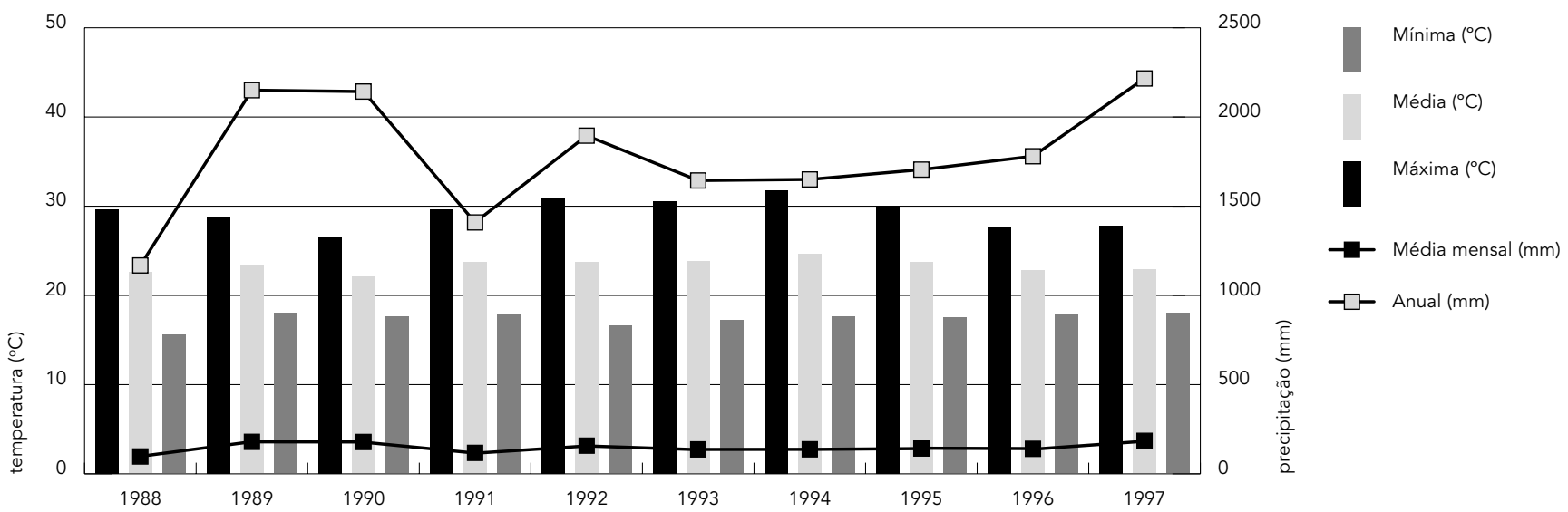

Tabela 1

Comparação quantitativa e da composição faunística de flebotomíneos coletados no mesmo galinheiro, na Fazenda Palmital, Município de Terra Boa, Paraná, Brasil, de novembro de 1988 a abril de 1990 e de janeiro a dezembro de 1997.

\begin{tabular}{|c|c|c|c|c|}
\hline Espécie/Ano & $88 / 90 *$ & $\%$ & 97 ** & $\%$ \\
\hline Lutzomyia migonei & 32.038 & 51,6 & 329 & 6,0 \\
\hline Lutzomyia whitmani & 20.260 & 32,6 & 4.930 & 89,8 \\
\hline Lutzomyia pessoai & 2.674 & 4,3 & 9 & 0,2 \\
\hline Lutzomyia firmatoi & 2.254 & 3,6 & - & - \\
\hline Lutzomyia fischeri & 1.883 & 3,0 & 58 & 1,1 \\
\hline Lutzomyia neivai & 1.090 & 1,7 & 159 & 2,9 \\
\hline Lutzomyia cortelezzii & 562 & 0,9 & - & - \\
\hline Lutzomyia shannoni & 435 & 0,7 & 1 & - \\
\hline Lutzomyia monticola & 256 & 0,4 & 2 & - \\
\hline Lutzomyia ferreirana & 149 & 0,2 & - & - \\
\hline Brumptomyia brumpti & 6 & - & 1 & - \\
\hline Lutzomyia misionensis & 4 & - & - & - \\
\hline Brumptomyia nitzulescui & 2 & - & - & - \\
\hline Lutzomyioa edwardisi & 1 & - & - & - \\
\hline Lutzomyia spp. & 446 & - & - & - \\
\hline Total & $62.060^{*}$ & - & $5.489 * \star$ & - \\
\hline Média Horária & 287 & - & 19 & - \\
\hline
\end{tabular}

* Flebotomíneos coletados das $21 \mathrm{~h}$ às $3 \mathrm{hs}$, em um total de 216 horas (galinheiro G1). ** Flebotomíneos coletados das $21 \mathrm{~h}$ às $24 \mathrm{~h}$, em um total de 288 horas (galinheiro G).

\section{Resultados}

As oito espécies de flebotomíneos coletadas foram: Brumptomyia brumpti (Larousse, 1920), Lutzomyia (Pintomyia) fischeri (Pinto, 1926), Lutzomyia migonei (França, 1920), Lutzomyia monticola (Costa Lima, 1932), Lutzomyia neivai (Pinto, 1926), Lutzomyia (Pintomyia) pessoai (Coutinho \& Barretto, 1940), Lutzomyia (Psathyromyia) shannoni (Dyar, 1929), Lutzomyia (Nyssomyia) whitmani (Antunes \& Coutinho, 1939).

Na Tabela 1, nota-se nitidamente que a média horária (MH) de 287 (62.060/216) flebotomíneos coletados em 1988/1989 foi muito superior à MH 19 (5.489/288) de 1997. Observase, também, que o número de espécies (14) coletado no primeiro período foi maior do que no segundo (oito espécies). No período de 1988 a 1990, observou-se o predomínio de L. migonei $(51,6 \%)$ sobre as demais espécies. Houve uma diferença de $19 \%$ entre a primeira (L. migonei) e a segunda (L. whitmani) espécie de maior prevalência. Nas coletas feitas em 1997, prevaleceu $L$. whitmani $(89,8 \%)$ com uma diferença de $83,8 \%$ em relação a $L$. migonei, que foi a segunda espécie mais prevalente $(6,0 \%)$. Nota-se, ainda, que, no período de 1988 a 1990, L. pessoai era a terceira espécie predominante, ocupando em 1997 o quinto lugar em prevalência. L. neivai, que foi a sexta espécie mais prevalente $(1,7 \%)$ em 1988/1990, em 1997 foi a terceira $(2,9 \%)$. L. fischeri, que era a quinta espécie pre- 
Flebotomíneos coletados com armadilhas de Falcão em galinheiro (G1), das 21h às 24h, na Fazenda Palmital, Município de Terra Boa, de janeiro a dezembro de 1997

\begin{tabular}{|c|c|c|c|c|c|c|c|c|c|c|c|c|c|c|}
\hline Espécie/Mês & 01 & 02 & 03 & 04 & 05 & 06 & 07 & 08 & 09 & 10 & 11 & 12 & Total & $\%$ \\
\hline L. whitmani & 38 & 386 & 698 & 102 & 37 & 9 & - & 318 & 64 & 1 & 2.075 & 1.202 & 4.930 & 89,8 \\
\hline L. migonei & 1 & 55 & 24 & 17 & - & 1 & - & 10 & 8 & 1 & 132 & 80 & 329 & 6,0 \\
\hline L. neivai & 1 & 22 & 22 & 7 & 2 & - & - & 13 & - & & 47 & 45 & 159 & 2,9 \\
\hline L. fischeri & - & 12 & 7 & 4 & - & - & - & - & - & - & 19 & 16 & 58 & 1,1 \\
\hline L. pessoai & - & 2 & 1 & 1 & 1 & - & - & - & - & - & 1 & 3 & 9 & - \\
\hline L. monticola & - & - & 1 & - & - & - & - & - & - & - & - & 1 & 2 & - \\
\hline L. shannoni & - & - & 1 & - & - & - & - & - & - & - & - & - & 1 & - \\
\hline B. brumpti & - & - & 1 & - & - & - & - & - & - & - & - & - & 1 & - \\
\hline Total & 40 & 477 & 755 & 131 & 40 & 10 & - & 341 & 72 & 2 & 2.274 & 1.347 & 5.489 & \\
\hline
\end{tabular}

dominante $(3,0 \%)$, é agora a quarta $(1,1 \%)$. As espécies L. firmatoi, L. cortelezzii, L. misionensis, L. edwardsi, L. ferreirana e Brumptomyia nitzulescui não foram coletadas em 1997.

Os flebotomíneos coletados de novembro a março representaram 89,1\% (4.893 exemplares) do total coletado, e L. whitmani prevaleceu sobre as outras espécies em todos os meses do ano de 1997 (Tabela 2).

\section{Discussão}

Os resultados deste trabalho corroboram observações de outros autores de que as alterações ambientais e o uso de inseticidas alteram também a biodiversidade da fauna, o tamanho da população e a proporção das espécies de flebotomíneos em relação à dominância (Forattini, 1960; Teodoro, 1995; Teodoro \& Kühl, 1997; Teodoro et al., 1998a).

Os resultados mostram que a quantidade de flebotomíneos coletada no galinheiro utilizado nesta investigação foi pequena comparada à coletada anteriormente no mesmo galinheiro (Teodoro et al. 1993b), onde estes autores coletaram 62.060 flebotomíneos com armadilhas de Falcão, entre $21 \mathrm{~h}$ e $3 \mathrm{~h}$. No peridomicílio de uma colônia de casas na Fazenda Jussara, município de Jussara, das $21 \mathrm{~h}$ às $24 \mathrm{~h}$, foram coletados 64.335 flebotomíneos, também com armadilhas de Falcão (Teodoro, 1995). Este número representou $39,2 \%$ do total de flebotomíneos coletados entre $21 \mathrm{~h}$ e $3 \mathrm{~h}$, na Fazenda Jussara. Pressupõe-se, portanto, que na Fazenda Palmital (Teodoro et al., 1993b) teríamos no mínimo o mesmo percentual de flebotomíneos coletados das $21 \mathrm{~h}$ às $24 \mathrm{~h}$.
Anteriormente, foi observada a tendência de L. whitmani prevalecer sobre as demais espécies, pois, nos meses de março, maio e junho de 1988 e janeiro, fevereiro e abril de 1990, este flebotomíneo foi mais freqüente que os outros (Teodoro et al., 1993b). Estes autores verificaram que a prevalência de L. migonei deveu-se às coletas feitas no galinheiro utilizado no presente trabalho (R1G no período 1988/1990). Verificou-se ainda que de 70.304 flebotomíneos coletados no peridomicílio 62.060 (88,3\%) eram provenientes do galinheiro R1G. Destes, 32.038 (51,6\%) eram representados por L. $m i$ gonei e 20.260 (32,6\%) por L. whitmani. Agora, no ambiente com maior grau de antropia, $L$. whitmani suplantou de vez as demais espécies em todos os meses que foram coletados flebotomíneos em 1997.

A coleta de maior número de flebotomíneos de novembro a março também foi observada anteriormente (Teodoro et al., 1993b), coincidindo com o período do ano em que a pluviosidade e a temperatura são mais elevadas.

Acredita-se que o corte de árvores ao redor do galinheiro somado às sucessivas desinsetizações exerceram maior influência na relação de dominância, no tamanho da população e na biodiversidade da fauna de flebotomíneos, pois, de 1988 a 1997, as condições meteorológicas não sofreram grandes oscilações (Figura 2). 


\section{Agradecimentos}

À Cia. Melhoramentos Norte do Paraná, com sede no município de Jussara, Paraná, pelas facilidades que proporcionou para a realização deste trabalho; à Sra. Rosalina Moretti Prina e Maycon Luiz Prina, que colaboraram nas coletas de flebotomíneos; e ao Sr. Carmelindo Colato, desenhista do Departamento de Engenharia Civil da Universidade Estadual de Maringá.

\section{Referências}

AGUIAR, G. M.; VILELA, M. L.; FERREIRA, V. A. \& SANTOS, T. G., 1989. Ecologia dos flebótomos em recente foco ativo de leishmaniose tegumentar no norte do Estado do Paraná (Diptera, Psychodidae, Phlebotominae). Memórias do Instituto Oswaldo Cruz, 84:7-8.

CAT, I.; LUZ, E.; BORBA, A. A.; CASSILHA, A.; COSTA, P. \& MARTINS, F. I., 1974. Leishmaniose visceral autóctone no oeste paranaense. Anais da Faculdade de Medicina da Universidade Federal do Paraná,16/17:27-35.

CONSOLIM, J.; LUZ, E. \& TORRES, P. B., 1990. Flebótomos da área do reservatório da hidroelétrica de Itaipu, Estado do Paraná, Brasil (Diptera, Psychodidae). Cadernos de Saúde Pública, 6:86-89.

FALCÃO, A. R., 1981. Um novo modelo de armadilha luminosa de sucção para pequenos insetos. $\mathrm{Me}$ mórias do Instituto Oswaldo Cruz, 76:303-305.

FORATTINI, O. P., 1960. Novas observações sobre a biologia de flebótomos em condições naturais (Diptera, Psychodidae), Arquivos da Faculdade Hig. de São Paulo, 25:209-215.

MARCONDES, C. B., 1996. A redescription of Lutzomyia (Nyssomyia) intermedia (Lutz \& Neiva, 1912), and ressurection of L. neivai (Pinto, 1926) (Diptera, Psychodidae, Phlebotominae). Memórias do Instituto Oswaldo Cruz, 91:457-462.

MARCONDES, C. B.; LOZOVEI, A. L. \& VILELA, J. H., 1998. Distribuição geográfica de flebotomíneos do complexo Lutzomyia intermedia (Lutz \& Neiva, 1912). Revista da Sociedade Brasileira de Medicina Tropical, 31:51-58.

ROBERTO, A. C. B. S.; LIMA, A. P.; PEIXOTO, P. R.; MISUTA, N. M.; FUKUSHIGUE, Y.; FERREIRA, M. E. M. C.; NERILO-SOBRINHO, A.; SILVEIRA, T. G. V. \& TEODORO, U., 1997. Avaliação da terapia com antimoniato de N-Metil glucamina e de notificação de leishmaniose tegumentar. Anais Brasileiros de Dermatologia, 72:129-136.

TEODORO, U., 1995. Características Ecológicas de Flebotomíneos (Diptera, Psychodidae) em Habitats Antrópicos, Município de Jussara, Paraná, Brasil. Tese de Doutorado, Curitiba: Faculdade de Ciências Biológicas/Entomologia, Universidade Federal do Paraná.
TEODORO, U. \& KÜHL, J. B., 1997. Interação flebotomíneos, animais domésticos e dominância de Lutzomyia (Nyssomyia) intermedia (Lutz \& Neiva, 1912) em área com alto grau de antropia, sul do Brasil. Revista de Saúde Pública, 31:512-516.

TEODORO, U.; LA-SALVIA-FILHO., V.; LIMA, E. M.; SPINOSA, R. P.; BARBOSA, O. C.; FERREIRA, M. E. M. C. \& SILVEIRA, T. G. V., 1993a. Flebotomíneos em área de transmissão de leishmaniose tegumentar na região norte do Estado do Paraná Brasil: Variação sazonal e atividade noturna. $R e$ vista de Saúde Pública, 27:190-194.

TEODORO, U.; LA-SALVIA-FILHO, V.; LIMA, E. M.; SPINOSA, R. P.; BARBOSA, O. C.; FERREIRA, M. E. M. C. \& LONARDONI, M. V. C., 1993b. Observações sobre o comportamento de flebotomíneos em ecótopos florestais e extraflorestais, em área endêmica de leishmaniose tegumentar americana, no norte do Estado do Paraná, sul do Brasil. Revista de Saúde Pública, 27: 242-249.

TEODORO, U.; GALATI, E. A. B.; KÜHL, J. B.; LOZOVEI, A. L. \& BARBOSA, O. C., 1998a. Controle de flebotomíneos com DDT, em área endêmica de leishmaniose tegumentar no Estado do Paraná, sul do Brasil. Brazilian Archives of Biology and Tecnology, 41:359-364.

TEODORO, U.; KÜHL, J. B.; RODRIGUES, M.; SANTOS, E. S.; SANTOS, D. R. \& MARÓSTICA, L. M. F., 1998b. Flebotomíneos coletados em mata remanescentes e abrigos de animais silvestres de zoológico no perímetro urbano de Maringá, sul do Brasil. Estudo preliminar. Revista da Sociedade Brasileira de Medicina Tropical, 31:517-522. 